\title{
LncRNA-FKBP1C regulates muscle fiber type switching by affecting the stability of MYH1B
}

\author{
Jia-ao Yu ${ }^{1,2,3}$, Zhijun Wang ${ }^{1,2,3}$, Xin Yang ${ }^{1,2,3}$, Manting Ma ${ }^{1,2,3}$, Zhenhui Li ${ }^{1,2,3}$ and Qinghua Nie $\mathbb{B}^{1,2,3}$
}

\begin{abstract}
Long non-coding RNAs (IncRNAs) are well-known to participate in a variety of important regulatory processes in myogenesis. In our previous RNA-seq study (accession number GSE58755), we found that InCRNA-FKBP1C was differentially expressed between White Recessive Rock (WRR) and Xinghua (XH) chicken. Here, we have further demonstrated that IncRNA-FKBP1C interacted directly with MYH1B by biotinylated RNA pull-down assay and RNA immunoprecipitation (RIP). Protein stability and degradation experiments identified that IncRNA-FKBP1C enhanced the protein stability of MYH1B. Overexpression of InCRNA-FKBP1C inhibited myoblasts proliferation, promoted myoblasts differentiation, and participated in the formation of skeletal muscle fibers. LncRNA-FKBP1C could downregulate the fast muscle genes and upregulate slow muscle genes. Conversely, its interference promoted cell proliferation, repressed cell differentiation, and drove the transformation of slow-twitch muscle fibers to fast-twitch muscle fibers. Similar results were observed after knockdown of the MYH1B gene, but the difference was that the MYH1B gene had no effects on fast muscle fibers. In short, these data demonstrate that IncRNA-FKBP1C could bound with MYH1B and enhance its protein stability, thus affecting proliferation, differentiation of myoblasts and conversion of skeletal muscle fiber types.
\end{abstract}

\section{Introduction}

Skeletal muscle is one of the most dynamic tissues involved in a variety of biological processes ${ }^{1}$, and the growth and development of skeletal muscle are essential for maintaining skeletal muscle function ${ }^{2}$. Skeletal muscle dysfunction can lead to a variety of human muscle diseases, including muscle dystrophy, type 2 diabetes, cardiomyopathic disease, and other metabolic disorders ${ }^{3-7}$. Skeletal muscle is composed of various muscle fibers types [including type I and type II (IIa, IIb, and IIx) muscle fibers] that are different in function, biochemical characteristics, and morphological characteristics ${ }^{8-10}$. Type I

\footnotetext{
Correspondence: Qinghua Nie (nqinghua@scau.edu.cn)

${ }^{1}$ State Key Laboratory for Conservation and Utilization of Subtropical Agrobioresources \& Lingnan Guangdong Laboratory of Agriculture, College of Animal Science, South China Agricultural University, Guangzhou, Guangdong, China

${ }^{2}$ Guangdong Provincial Key Lab of Agro-Animal Genomics and Molecular Breeding, and Key Laboratory of Chicken Genetics, Breeding and Reproduction, Ministry of Agriculture, Guangzhou, China

Full list of author information is available at the end of the article

These authors contributed equally: Jia-ao Yu, Zhijun Wang

Edited by Ivano Amelio
}

fibers have higher mitochondrial and lower glycogen and glucose contents, while type II fibers have a higher glycolytic capacity. Previous research has reported that the ratio of muscle fiber types could affect meat quality ${ }^{11-13}$. In addition, skeletal muscle is dynamic muscle tissue, the fast muscle fiber subtype (type II) and the slow muscle fiber subtype (type I) can mutually transform regulated by noncoding RNAs ${ }^{2,14,15}$.

In the past, lncRNAs genes had been regarded as junk DNA. However, with the continuous advancement of sequencing technology, IncRNA have been found to participate in a variety of important regulatory processes ${ }^{16-19}$. LncRNAs are a type of RNA molecule whose transcript length exceeds $200 \mathrm{nt}$ and does not encode protein in common $^{20,21}$. Recent studies have demonstrated that lncRNAs play an important regulatory role in the skeletal muscle growth, development ${ }^{22-24}$, and muscle fibers conversion of livestock. Moreover, it has been reported that IncRNA can be an important participant in DNA methylation regulation ${ }^{25,26}$. LncRNAs have been reported to carry out diverse functions in trans ${ }^{19,27}$. Researchers 
found that non-coding RNA could play a role by binding to proteins and affecting their stability ${ }^{28}$.

$M Y H 1 B$, also known as $M Y H 3$, is mainly expressed in various developmental stages, including the embryonic stage of skeletal muscle ${ }^{29}$. In addition, coding mutations in the $M Y H 3$ gene can cause muscle development disorders in humans $^{30,31}$. MYH3 gene can regulate not only muscle fiber type conversion but also adipogenesis in skeletal muscle ${ }^{32}$. However, few reports have been made about $M Y H 1 B$ in poultry skeletal muscle development.

In our previous study, we found that the IncRNAFKBP1C was differentially expressed between breast muscle tissues of Recessive White Rock (WRR) and Xinghua Chickens $(\mathrm{XH})$. This result suggested that
lncRNA-FKBP1C might affect chicken muscle growth. In this study, we explored the regulation of lncRNA-FKBP1C in chicken skeletal muscle development. We concluded that IncRNA-FKBP1C could bind to MYH1B and play a role by affecting its stability. LncRNA-FKBP1C also could regulate myoblast proliferation and differentiation, and induce the slow-twitch muscle phenotype.

\section{Results}

cDNA sequence, protein-encoding ability, and expression level of IncRNA-FKBP1C

We used RACE analysis to obtain the 5' and 3' ends of IncRNA-FKBP1C (Fig. 1A). We used the Coding Potential Calculator to predict the protein-encoding ability of
A

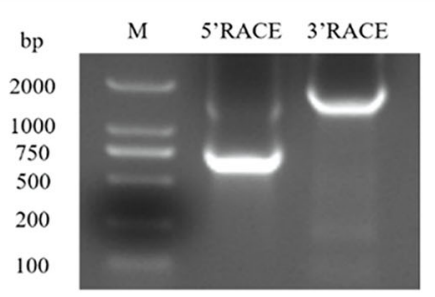

B

\begin{tabular}{|l|l|l|l|}
\hline \multirow{4}{*}{ HOMOLOGY FEATURES } & HIT NUM & 0 & \\
\cline { 2 - 4 } & HIT SCORE & 0.0 & \\
\cline { 2 - 4 } & FRAME SCORE & 0.0 & \\
\hline \multirow{3}{*}{ ORF_FRAMEFINDER } & COVERAGE & $55.10 \%$ & \\
\cline { 2 - 4 } & LOG-ODDS SCORE & 108.59 & \\
\cline { 2 - 3 } & TYPE & Partial & \\
\hline \multirow{2}{*}{ legend: non-coding coding } & \multicolumn{3}{|l}{} \\
\hline
\end{tabular}

C

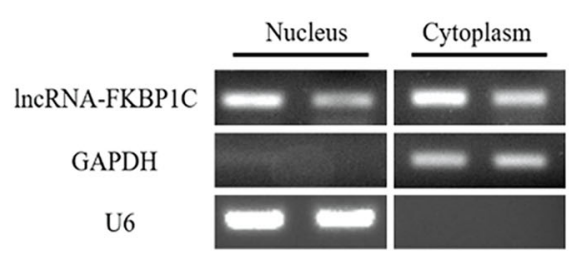

$\mathbf{E}$

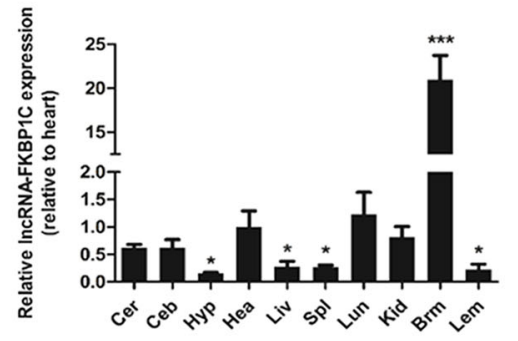

$\mathbf{F}$

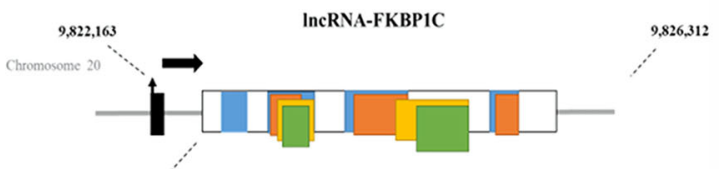

$9,823,275$

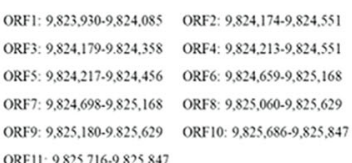

ORF 1 : $9.825,716-9.825,847$

Fig. 1 Identification of IncRNA-FKBP1C. A Results of 5' RACE and 3' RACE. M, DL2000 Marker; 5' RACE product, 690 bp; 3' RACE product, 1465 bp. B Analysis obtained from the Coding Potential Calculator (http://cpc.cbi.pku.edu.cn/) based on evolutionary conservation and ORF attributes. C LnCRNA-FKBP1C is localized in the cytoplasm and nucleus of chicken primary myoblasts. GAPDH and U6 serve as cytoplasmic and nuclear localization controls, respectively. D XH chicken tissue expression profiles of IncRNA-FKBP1C. Cer, cerebrum; Ceb, cerebellum; Hyp, hypothalamus; Pit, pituitary; Hea, heart; Liv, liver; Spl, spleen; Lun, lung; Kid, kidney; Brm, breast muscle; Lem, leg muscle. E Western blot analysis of the coding ability of IncRNA-FKBP1C. The possible ORF of IncRNA-FKBP1C was cloned into the eukaryotic expression vector pcDNA3.1-3xFlag. Untransfected DF-1 cells were used as a negative control (NC) and DF-1 cells transfected with pcDNA3.1-3xFlag- $\beta$-actin were used as a positive control. The upper panel shows the model target fragment in the pcDNA3.1-3xFlag vector. The lower left panel is the blot of ORFs 1-11, and the lower right panel is the blot of the NC and $\beta$-actin. $\mathbf{F}$ The location of 11 potential ORFs in InCRNA-FKBP1C. 11 potential ORFs are arranged in order according to their positions. Results are shown as mean \pm S.E.M. and the data are representative of at least three independent assays. Independent sample t-test was used to analyze the statistical differences between groups $\left({ }^{*} P<0.05,{ }^{* *} P<0.001\right)$. 


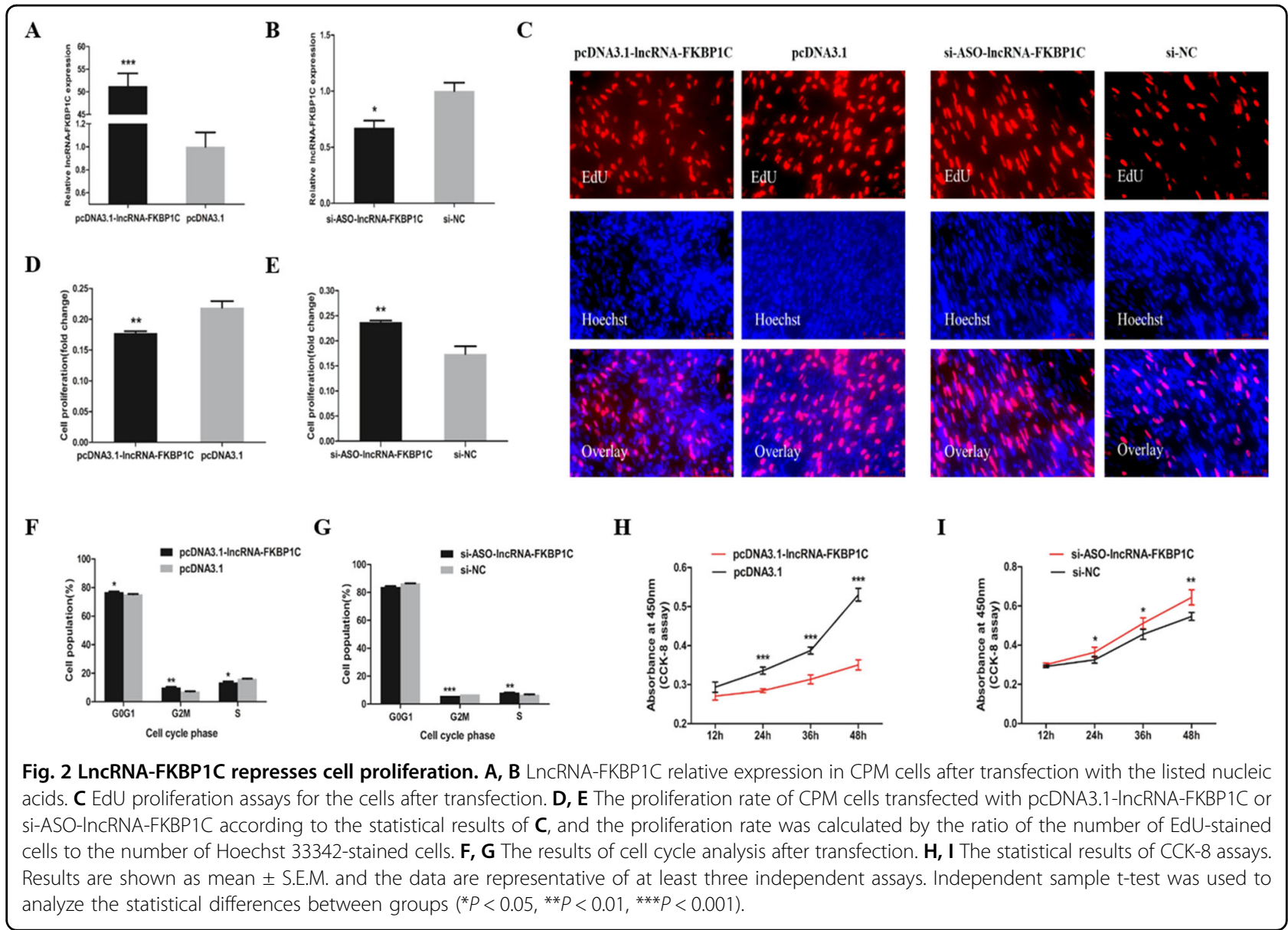

lncRNA-FKBP1C and the result suggested a high coding potential (Fig. 1B). We analyzed the protein-encoding ability of eleven potential ORFs of lncRNA-FKBP1C by Western blot to verify this prediction. We found that lncRNA-FKBP1C is a lncRNA without protein-encoding potential (Fig. 1E, F). The NCBI BLAST indicated that lncRNA-FKBP1C was 3, 038 bp long, located on Chromosome 20 and spanned from 9,823,275-9,826,312, and comprised an intron. We further investigated the subcellular localization of lncRNA-FKBP1C, and the RT-PCR results confirmed that it is an RNA molecule present in the cytoplasm and nucleus (Fig. 1C). We also measured lncRNA-FKBP1C expression from different tissues of 7week-old XH chickens using qRT-PCR, the results showed that lncRNA-FKBP1C was highly expressed in breast muscle (Fig. 1D).

LncRNA-FKBP1C represses the proliferation of myoblasts

To unveil the function of lncRNA-FKBP1C in myoblasts, we transfected the lncRNA-FKBP1C overexpression vector and its small interference RNA (siRNA) into the CPM cells to assess its effect on cell proliferation (Fig. 2A, B). According to the EdU assay, the overexpression of
lncRNA-FKBP1C significantly hampered cell proliferation in CPM (Fig. 2C, D) and after lncRNA-FKBP1C knockdown the results were opposite (Fig. 2C, E). LncRNAFKBP1C overexpression also significantly increased the number of G0/G1 cells, and the number of $S$ phase cells was lower than the control group. Conversely, the cell cycle changes showed the opposite trend with lncRNAFKBP1C interference (Fig. 2F, G). Moreover, we found that lncRNA-FKBP1C overexpression reduced cell viability by using the CCK-8 assay (Fig. 2H). After the IncRNAFKBP1C knockdown, more cells were detected than the control group (Fig. 2I). Collectively, these data revealed that IncRNA-FKBP1C represses the proliferation of myoblasts.

LncRNA-FKBP1C promotes the differentiation of myoblasts

The MyHC immunofluorescence staining results showed that IncRNA-FKBP1C overexpression significantly increased the area of myotubes (Fig. 3A, C), while knockdown of lncRNA-FKBP1C could prevent the formation of myotubes (Fig. 3B, C). GM stands for myoblasts in the proliferative stage, and DM1-DM6 stands for myoblasts that successfully induced 


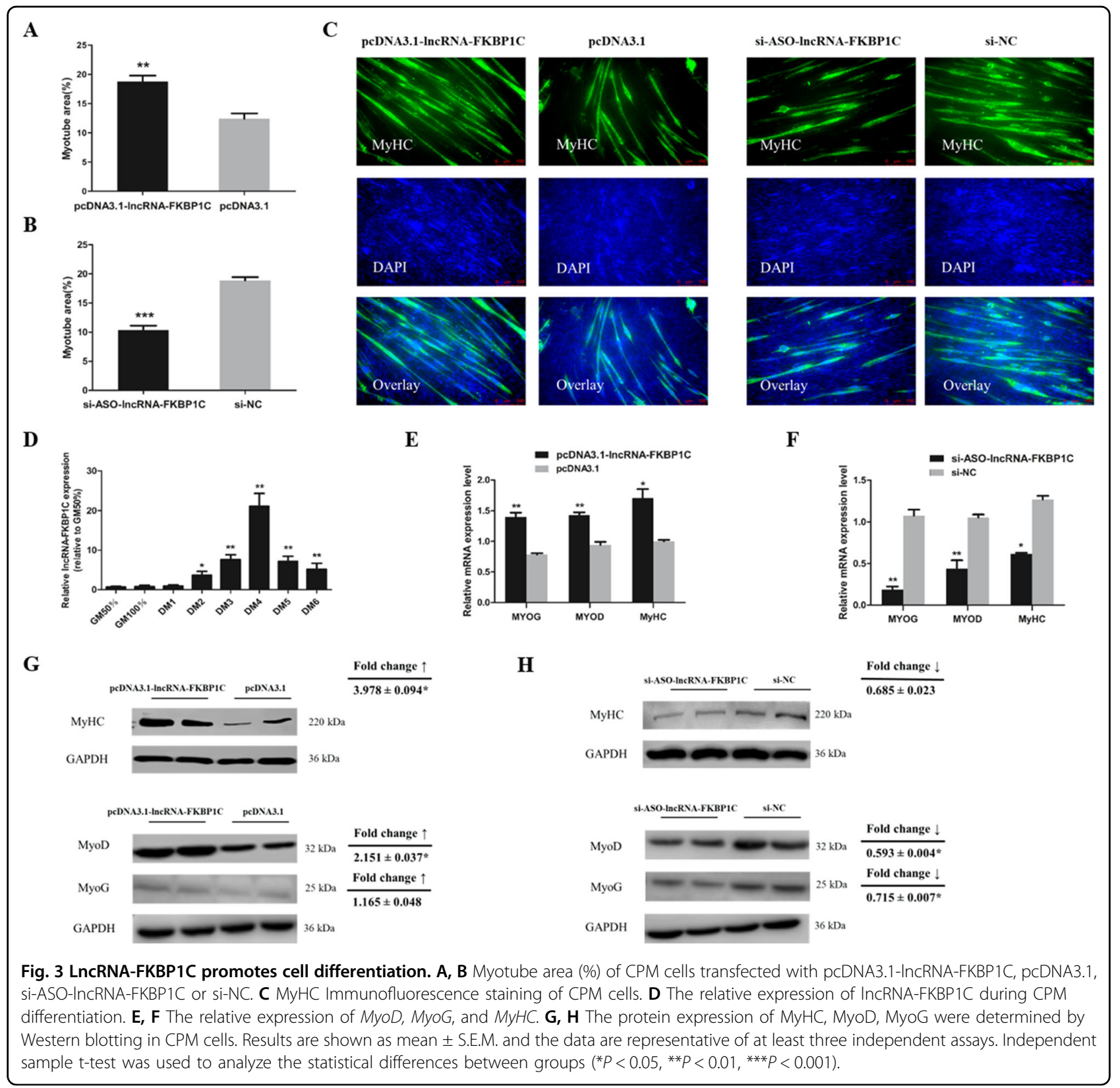

differentiation from day 1 to day 6 (at different time points). The expression of lncRNA-FKBP1C was gradually increased until DM4 and then decreased (Fig. 3D) during the induction of differentiation of myoblasts. After lncRNA-FKBP1C overexpression, we found that the expression of $M y o D, M y o G$, and $M y H C$ (myoblast differentiation marker genes) were remarkably increased. However, the knockdown of IncRNA-FKBP1C had an opposite effect on these genes (Fig. 3E, F). The protein level of MyHC, MyoD, MyoG were increased while transfected with lncRNA-FKBP1C overexpression vector by western blot analysis. However, after lncRNAFKBP1C interference, the results were the opposite
(Fig. 3G, H). In short, IncRNA-FKBP1C could promote myoblast differentiation.

\section{LncRNA-FKBP1C participates in the formation of skeletal muscle fibers}

Since lncRNA-FKBP1C was differentially expressed in breast and leg muscles of 7-week-old XH chickens, we suspected that IncRNA-FKBP1C may affect the conversion of skeletal muscle fiber types. So we detected the relative mRNA level of a series of fast muscle genes (including Wnt4, Tnnc2, Tnnt3, and $S r l$ ) and a series of slow muscle genes (including Sox6, Tnnc1, Tnni1, and Tnnt1) (Fig. 4A, B). After lncRNA-FKBP1C overexpression, the expression 


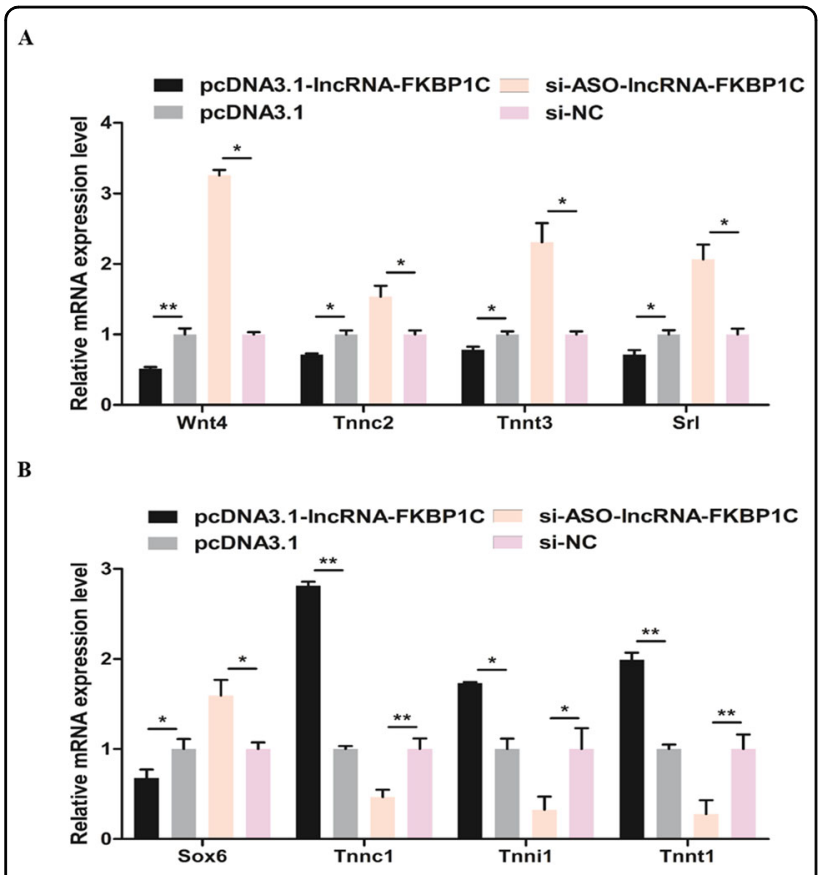

Fig. 4 LncRNA-FKBP1C participates in the formation of skeletal muscle fibers. A, B The relative expression of several fast muscle genes and slow muscle genes induced by IncRNA-FKBP1C overexpression and interference in CPM cells. Results are shown as mean \pm S.E.M. and the data are representative of at least three independent assays. Independent sample $t$-test was used to analyze the statistical differences between groups $\left({ }^{*} P<0.05,{ }^{* *} P<0.01\right)$.

of fast muscle genes was decreased significantly and the expression of slow muscle genes was increased. The results of IncRNA-FKBP1C interference showed that it could drove the transformation of slow-twitch muscle fibers to fast-twitch muscle fibers.

\section{LncRNA-FKBP1C interacts directly with MYH1B and enhances its protein stability}

To further explore the molecular mechanism of lncRNA-FKBP1C, we analyzed the localization of lncRNAFKBP1C and found that this lncRNA was expressed in the nucleus. Also, lncRNAs have been proven to regulate the binding proteins in trans ${ }^{19}$. Thus, we hypothesized that IncRNA-FKBP1C may function by regulating its RNAbinding proteins. To certify this hypothesis, we carried out a biotinylated RNA pull-down assay to identify proteins associated with lncRNA-FKBP1C in CPM cells. RNAbinding proteins were analyzed by silver staining and mass spectrometry (Fig. 5A). Next, we selected these five most abundant proteins (including MYH1B, MYH1A, TPM1, TPM3, and MYH11) from the mass spectrometry result to verify whether they could interact with lncRNA-FKBP1C. The results showed that MYH1B was predicted to have extremely high possibilities combined with lncRNAFKBP1C using catRAPID graphic (Fig. 5B). To further determine this specific interaction in vitro, we once again performed a biotinylated RNA pull-down assay to obtain the protein products by using biotinylated IncRNAFKBP1C with biotinylated antisense RNA as the negative control. MYH1B was detected by Western blot assay from the obtained protein products by biotinylated RNA pulldown assays in primary myoblasts (Fig. 5C). Meanwhile, this interaction was further verified with RNA immunoprecipitation (RIP) of MYH1B. As expected, MYH1B pulled down lncRNA-FKBP1C in CPM cell lysates, revealing lncRNA-FKBP1C could interact with MYH1B in vivo (Fig. 5D, E). To sum up, IncRNA-FKBP1C interacts specifically with MYH1B in CPM cells in vitro and in vivo.

Next, we tried to explore the influence of lncRNAFKBP1C on MYH1B. The relative mRNA expression of $M Y H 1 B$ and its protein level was detected after lncRNAFKBP1C overexpression or knockdown. qRT-PCR results indicated that lncRNA-FKBP1C did not affect MYH1B mRNA level (Fig. 5F). However, we found a significant upregulation of MYH1B protein with overexpression of lncRNA-FKBP1C in myoblasts, while lncRNA-FKBP1C knockdown showed the opposite trend (Fig. 5G). Based on these data, we thought that lncRNA-FKBP1C may bind to MYH1B to affect its biological function at the translational or posttranslational level. To verify these hypotheses, we used protein synthesis inhibitor cycloheximide (CHX) and proteasome inhibitor MG-132 to process the CPM cells that had overexpressed or knocked-down of IncRNAFKBP1C. CHX can decrease the expression of proteins by inhibiting protein synthesis and MG-132 mainly inhibits protein degradation by inhibiting ubiquitinationproteasome dependent degradation pathway. The western blot assays showed that overexpression of IncRNAFKBP1C slowed down a decrease in the expression of MYH1B proteins under the treatment of CHX compared with those in control groups, which meant that overexpression of lncRNA-FKBP1C counteracted the inhibitory effect of $\mathrm{CHX}$ on MYH1B protein (Fig. $5 \mathrm{H}$ ). At the same time, interference with lncRNA-FKBP1C aggravated the decline of MYH1B protein expression level (Fig. 5I). In addition, MG-132 rescued the inhibitory effect of lncRNAFKBP1C knockdown on MYH1B protein after $6 \mathrm{~h}$ of treatment (Fig. 5J), and overexpression of lncRNAFKBP1C under the treatment of MG-132 can increase the protein expression level of MYH1B (Fig. 5K). In summary, these data demonstrated that lncRNA-FKBP1C specifically interacts with MYH1B and upregulates its protein level by enhancing its protein stability.

\section{MYH1B regulates cell proliferation and differentiation of myoblast and also induces the slow-twitch muscle phenotype}

To investigate the function of $M Y H 1 B$, we detected the mRNA and protein expression of $M Y H 1 B$ after 


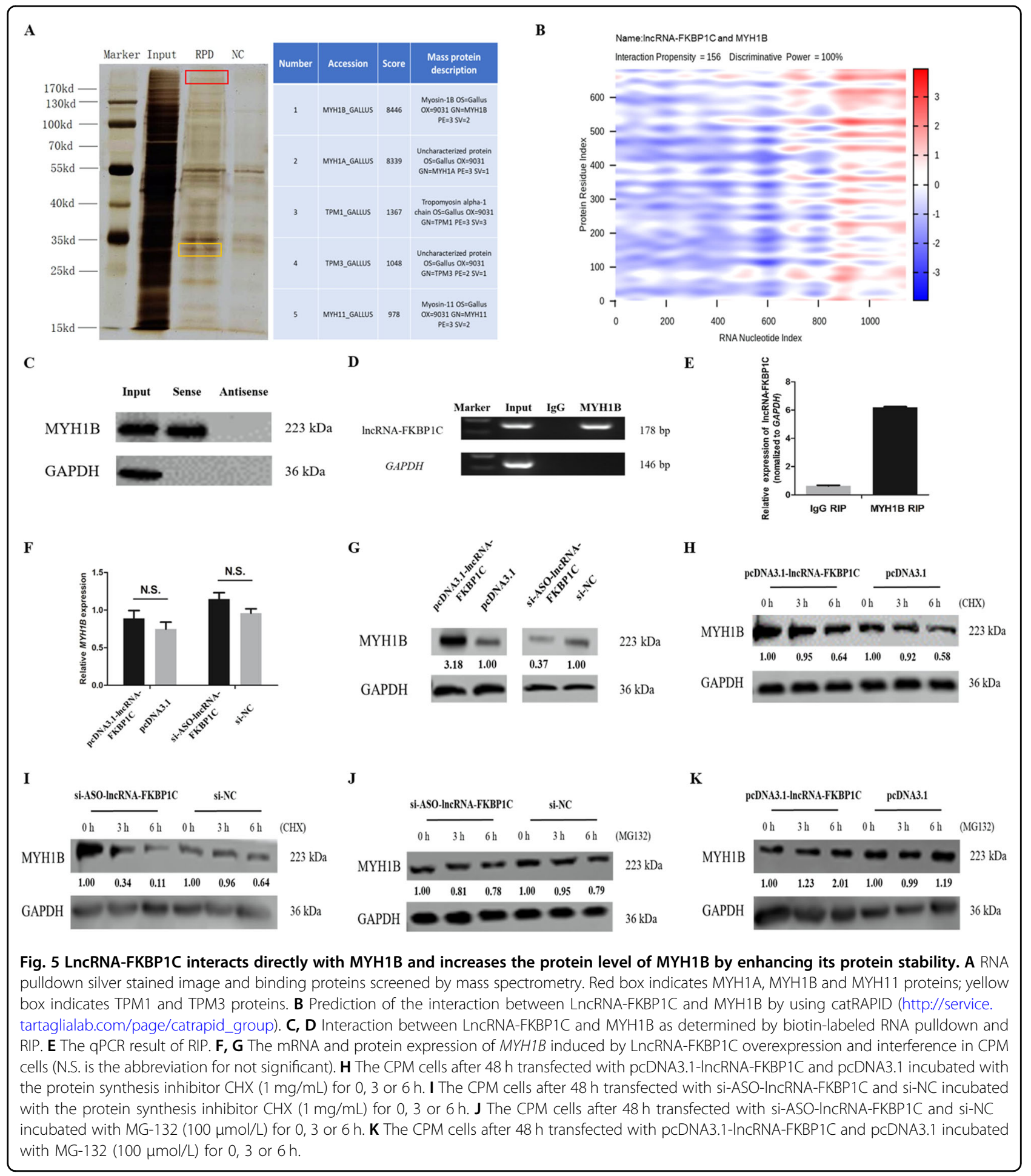

interference with $M Y H 1 B$ in primary myoblasts (Fig. 6A, B). $M Y H 1 B$ knockdown in myoblasts remarkably downregulated the relative mRNA expression of $M y o D$, $M y o G$, and $M y H C$ (Fig. 6C), and decreased the myotube area as showed in the $\mathrm{MyHC}$ immunofluorescence staining results (Fig. 6E, F). According to western blot analysis, the protein level of $\mathrm{MyHC}$ decreased after MYH1B knockdown (Fig. 6D). In CPM cells, the interference of $M Y H 1 B$ significantly increased the EdU stained positive cells (Fig. 6G, H). This also resulted in a significant decrease in the number of cells in G0/G1 phase, and a significant increase in the number of S-phase cells 


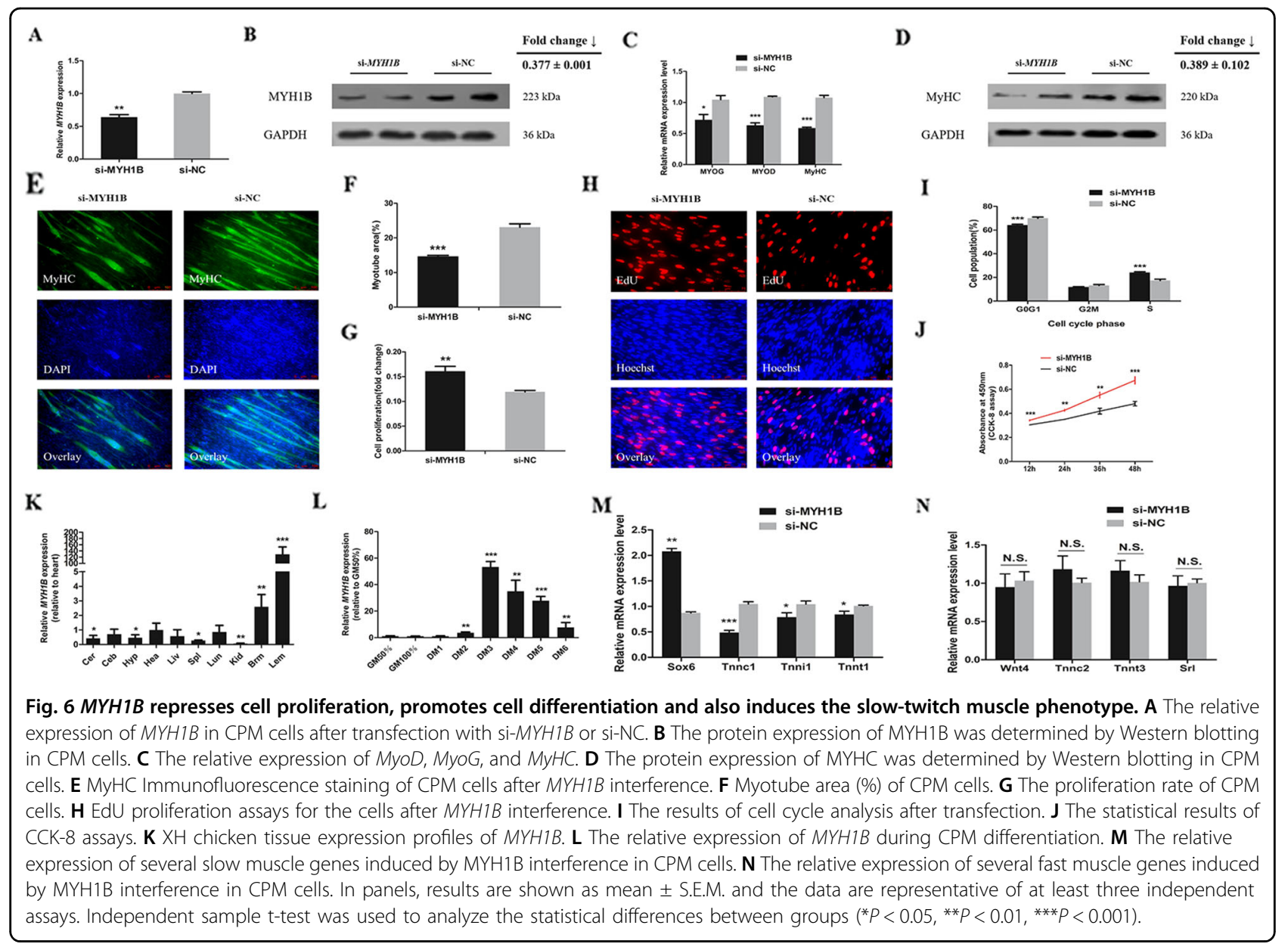

by flow cytometric analysis (Fig. 6I). Furthermore, the results of CCK-8 assay showed that $M Y H 1 B$ knockdown promoted cell proliferation (Fig. 6J). According to the tissue expression profiles and for $M Y H 1 B$, we found it was highly expressed in breast and leg muscles of 7-week-old $\mathrm{XH}$ chickens (Fig. 6K). The relative mRNA expression of $M Y H 1 B$ increased abruptly at DM3 and then decreased at the stage of proliferation and differentiation in CPM (Fig. 6L). As described above, these data demonstrated that $M Y H 1 B$ inhibited cell growth and proliferation and promoted cell differentiation of myoblast.

As $M Y H 3$ (called $M Y H 1 B$ in chickens) has been reported to influence the myofiber composition in mice, we examined the relative mRNA level of fast-type muscle fiber-associated genes (including Wnt4, Tnnc2, Tnnt3, and $S r l)$ and slow/type1/oxidative fiber associated genes (including Sox6, Tnnc1, Tnni1, and Tnnt1). By qRT-PCR analysis, the data revealed strongly decreased gene expression of slow muscle genes after interference MYH1B (Fig. 6M). However, knockdown $M Y H 1 B$ had no significant expression difference in fast muscle genes (Fig. $6 \mathrm{~N}$ ). Combined, we argue that $M Y H 1 B$ not only plays a vital role in myoblast development but also in the transformation of muscle fibers.

\section{LncRNA-FKBP1C influence muscle fiber conversion and hypertrophy in vivo}

To further prove that lncRNA-FKBP1C could affect the conversion of skeletal muscle fiber types in vivo, we performed lentiviral infection on the gastrocnemius muscles of 1-day-old chicks (including overexpression and interference of lncRNA-FKBP1C). Glycogen content was significantly decreased with overexpression of lncRNAFKBP1C, and the opposite result was shown in the interference lncRNA-FKBP1C group (Fig. 7A, H). Overexpression of IncRNA-FKBP1C in vivo inhibited the enzyme activities of lactate dehydrogenase (LDH) and significantly increased the enzyme activities of succinate dehydrogenase (SDH), while knockdown of lncRNAFKBP1C enhanced enzyme activities of LDH and decreased enzyme activities of SDH (Fig. 7B, I). The results of immunohistochemical staining of MYH1A and MYH7B showed that IncRNA-FKBP1C could drove the transformation of fast-twitch muscle fibers to slow-twitch 


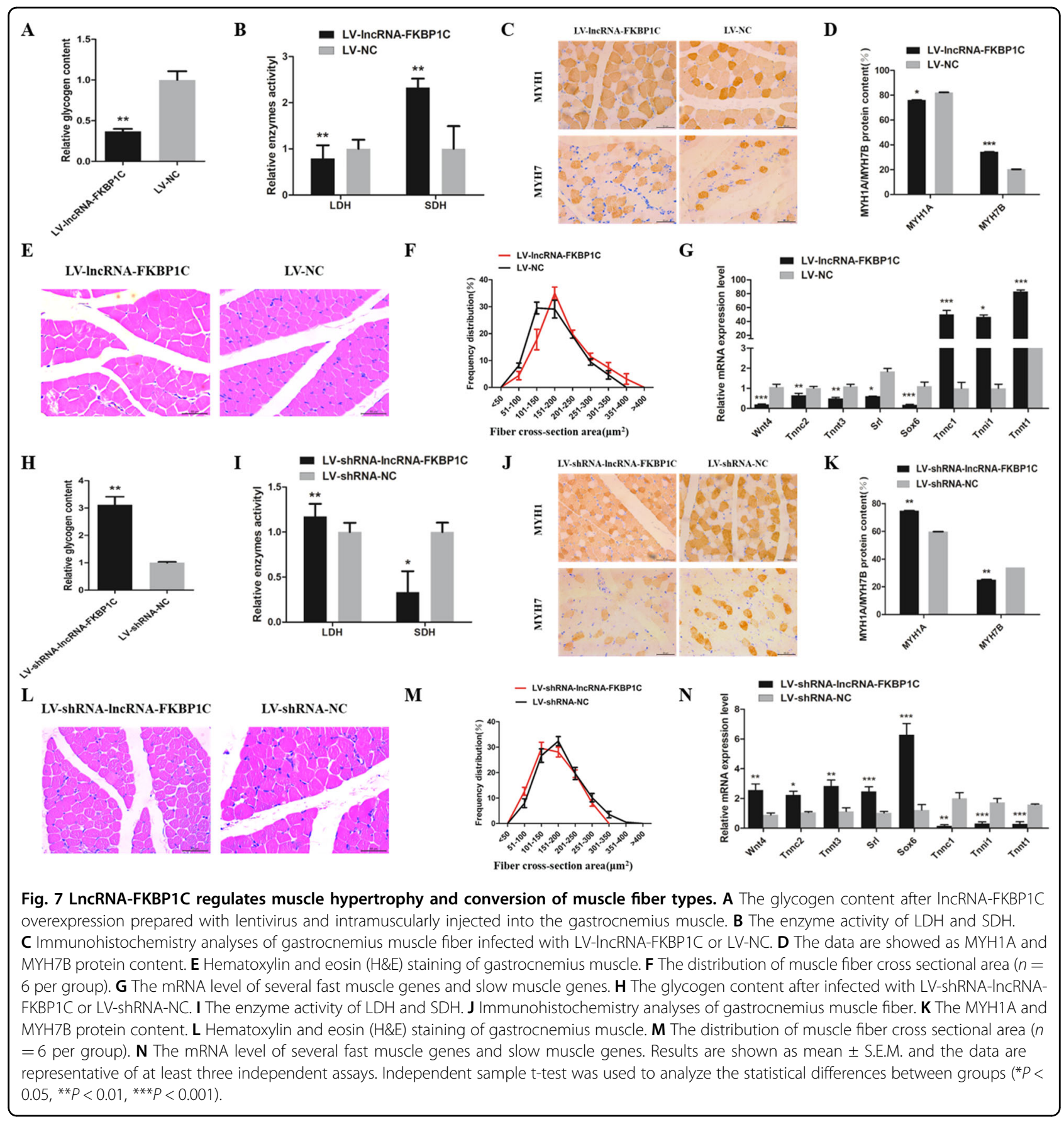

muscle fibers (Fig. 7C, D, J, and K). Besides, we detected the expression of fast-type and slow-type muscle fiber associated genes in vivo. We found that the results were consistent with those in the CPM cells (Fig. 7G, N). Moreover, the frequency distribution of muscle fiber cross-sectional area with overexpression and interference of lncRNA-FKBP1C showed that lncRNA-FKBP1C could promote muscle fiber hypertrophy (Fig. 7E, F, L, and M). We conclude that IncRNA-FKBP1C is associated with muscle fiber conversion and hypertrophy in vivo.

\section{Discussion}

It is well known that lncRNAs can act as an important regulatory factor in myogenesis ${ }^{22-24,33-38}$. For instance, lncRNA-SYISL was reported to function as a repressor of muscle development and play a vital role in muscle development mediated by PRC2 ${ }^{24}$. In this present study, we first reveal the function of IncRNA-FKBP1C in myoblast proliferation and differentiation. Also, lncRNAFKBP1C is involved in the formation of skeletal muscle fibers. Overexpression of lncRNA-FKBP1C represses cell 
proliferation and promotes cell differentiation in myoblasts while showing a down-regulation of fast muscle genes expression and up-regulation of slow muscle genes expression. To further explore the role of IncRNAFKBP1C in skeletal muscle fiber type conversion, we injected lentivirus into the living gastrocnemius muscle. All data showed that overexpression of IncRNA-FKBP1C could inhibit fast muscle fibers type formation and promote slow muscle fibers type formation. In summary, lncRNA-FKBP1C divers the transformation of fast-twitch muscle fibers to slow-twitch muscle fibers. Some reports suggest that the composition of muscle fiber types may influence meat quality by affecting the content of metabolites postmortem in livestock ${ }^{11-13,39,40}$, such as $\mathrm{pH}$, meat color, and drip loss ${ }^{41}$. Therefore, our findings can provide new ideas for improving poultry meat quality.

Some lncRNAs were reported to regulate neighboring gene expression in cis, while more and more lncRNAs were found leaving the transcription site and functioning in trans. Trans-acting lncRNAs may interact with and regulate the expression of proteins as a molecular $\operatorname{decoy}^{28,42,43}$. Therefore, we presume that IncRNAFKBP1C functions in trans. The lncRNA-FKBP1C was found to associate with MYH1B to enhance its protein stability. However, the underlying mechanisms of how IncRNA-FKBP1C regulates the ubiquitination or ubiquitination associated enzymes require further investigation.

$M Y H 3$ is indispensable in the development of skeletal muscle and heart. A lot of reports suggest that mutations of $M Y H 3$ can contribute to human bone or musclerelated diseases, such as Atrial septal defect (ASD), Sheldon-Hall syndrome (SHS), and Spondylocarpotarsal synostosis syndrome $(\mathrm{SCTS})^{44-46}$. In addition, recent research reported that $M Y H 3$ could promote slow muscle fibers but have no significant effect on fast muscle fibers in mice ${ }^{33}$. In our current study, we found that $M Y H 1 B$ could induce the slow-twitch muscle phenotype in chicken, as same as the function of $M Y H 3$ in mice. Also, $M Y H 1 B$ represses cell proliferation and promotes cell differentiation in myoblasts.

Collectively, the results of our study indicate that IncRNA-FKBP1C can interact directly with MYH1B to enhance its protein stability, thus influencing myoblast proliferation and differentiation, as well as regulating the transformation of skeletal muscle fiber types.

\section{Materials and methods}

\section{Ethics statement}

All animal experiments in this study were conducted in strict accordance with the regulations for the Administration of Laboratory Animals of Guangdong Province. These experiments (approval number: SCAU\#2020C030) were carried out under the approval of the Institutional Animal Care and Use Committee at the South China
Agricultural University (Guangzhou, China). We made every effort to reduce the suffering of animals.

\section{Experimental animals and tissues}

Four 7-week-old Xinghua female chickens were received from the Zhicheng Poultry Breeding Co., Ltd. (Guangdong, China). The tissues were collected, quickly frozen into liquid nitrogen, and then stored at $-80{ }^{\circ} \mathrm{C}$, including the cerebrum, cerebellum, hypothalamus, heart, liver, spleen, lung, kidney, breast muscle, and leg muscle.

\section{Cell culture}

The chicken primary myoblasts (CPM) were isolated from leg muscle at the gestational age of 11 (Zhuhai Yuhe Co., Ltd., China). First, the leg muscle tissues were cut into pieces after removing the skin and bone and then digested with pancreatin containing $0.25 \%$ EDTA for 15-20 min at $37^{\circ} \mathrm{C}$. Next, the digestion was terminated with Roswell Park Memorial Institute (RPMI)-1640 medium (Gibco, USA) containing 20\% fetal bovine serum (FBS, Gibco), and the digested tissue fluid was filtered through a sterile filter. The single cells were collected by centrifugation at $1500 \mathrm{r} / \mathrm{min}$ for $5 \mathrm{~min}$. Afterward, chicken primary myoblasts were obtained by the "differential adhesion method". We cultured CPM cells in RPMI-1640 medium (Gibco, USA) with 20\% FBS, 0.2\% penicillin, $0.2 \%$ streptomycin, and then reduced FBS in the medium to $5 \%$ to induce differentiation.

DF-1 cells were from previously frozen chicken embryo fibroblasts, cultured in Dulbecco's modified Eagle's medium (DMEM; Gibco) with 10\% FBS, $0.2 \%$ penicillin, $0.2 \%$ streptomycin. All cells were cultured at $37^{\circ} \mathrm{C}$ in a $5 \% \mathrm{CO} 2$ cell incubator.

\section{RNA isolation, complementary DNA(cDNA) synthesis, and real-time (RT) PCR analysis}

Total RNA was extracted from tissues or cells using RNAiso plus reagent (TaKaRa, Japan). After measuring the optical density at a Nanodrop 2000c spectrophotometer (Thermo, Waltham, MA, USA), the qualified total RNA was stored at $-80^{\circ} \mathrm{C}$. The cytoplasmic and nuclear cell lysates were obtained by using a PARIS Kit (Ambion, Life Technologies, USA) according to the manufacturer's protocol. cDNA synthesis was obtained by using a PrimeScript RT Reagent Kit with gDNA Eraser (Perfect Real Time) (Takara, Japan). Real-time quantitative PCR (qRT-PCR) reactions were performed on a QuantStudio 5 Real-Time PCR Systems (Thermo Fisher, Waltham, MA, USA) by using an iTaq Universal SYBR Green Supermix Kit (Bio-Rad Laboratories Inc., USA). qRT-PCR data were analyzed by the comparative $2^{-\Delta \Delta C T}$ method $^{47}$. The primers used for qRT-PCR in this study were listed in Supplementary Table S1. 


\section{Rapid-amplification of CDNA ends (RACE)}

The partial lncRNA-FKBP1C sequence was obtained from our previous lncRNA-seq data (accession number GSE58755). RACE PCR was carried out to obtain the fulllength sequence of the lncRNA-FKBP1C. Total RNA from breast muscle tissue was used as the template for nestedPCR reactions using a SMARTer RACE cDNA Amplification Kit (Clontech, Osaka, Japan), according to the manufacturer's instructions. The products of the RACE PCR were cloned into the pMD18-T cloning vector (PCR Cloning Kit; Fermentas, Glen Burnie, MD, USA) and sequenced by Tsingke Biotech (Guangzhou, China). Primers used for RACE PCR were listed in Supplementary Table S2.

\section{Plasmid construction and transfection}

Eleven ORFs of lncRNA-FKBP1C were amplified and cloned into pcDNA3.1-3xFlag (SiDanSai, Shanghai, China), and pcDNA3.1-3xFlag- $\beta$-actin was used as a positive control. All primers used in this study were designed using Premier Primer 5.0 software (Premier Biosoft International, Palo Alto, CA, USA) or OLIGO Primer Analysis Software Version 7 (Molecular Biology Insights, USA), and synthesized by Tsingke Biotech (Guangzhou, China). Primers used for vector construction were listed in Supplementary Table S2.

Lipofectamine 3000 reagent (Invitrogen, USA) was used for transfections in this study, according to the manufacturer's protocol. To interfere with lncRNA-FKBP1C, silncRNA-FKBP1C and ASO-lncRNA-FKBP1C were cotransfected into CPM, named as si-ASO-lncRNA-FKBP1C in this study. The transfection concentrations of siRNAs and ASO were $100 \mathrm{nM}$. All the siRNAs and ASO were obtained from RiboBio (Guangzhou, China) and the sequences were shown in Supplementary Table S3.

\section{EdU (5-ethynyl-2'-deoxyuridine) assay}

At $48 \mathrm{~h}$ after transfection, the CPM cells were incubated for $2 \mathrm{~h}$ in $50 \mu \mathrm{M}$ 5-ethynyl-2'-deoxyuridine (EdU; RiboBio, China). And then the cells were fixed in $4 \%$ paraformaldehyde for $30 \mathrm{~min}$ and stained with a C10310 EdU Apollo In Vitro Imaging Kit (RiboBio, China). The ratio of the number of EdU-stained cells to the number of Hoechst 33342-stained cells was determined using images of three randomly selected fields obtained with a fluorescence microscope (TE2000-U; Nikon, Japan).

\section{Flow cytometric analysis}

At $48 \mathrm{~h}$ after transfection, the CPM cells were collected and fixed in $70 \%$ ethanol overnight at $-20{ }^{\circ} \mathrm{C}$. The flow cytometry analysis was performed on a BD AccuriC6 flow cytometer (BD Biosciences, USA) by using a Cell Cycle Analysis Kit (Thermo Fisher Scientific, Waltham, MA, USA) and data were processed using FlowJo7.6 software (Treestar Incorporated, Ashland, OR, USA).

\section{CCK-8 assays}

The CPM cells cultured in a 96-well plate were transfected, and the proliferation of the cell culture was monitored at $12 \mathrm{~h}, 24 \mathrm{~h}, 36 \mathrm{~h}$, and $48 \mathrm{~h}$ using the TransDetect CCK (TransGen Biotech, Beijing, China). The cells were added $10 \mu \mathrm{L}$ of CCK solution and incubated in $1 \mathrm{~h}$, using a Model 680 Microplate Reader (Bio-Rad) to measure the absorbance at $450 \mathrm{~nm}$.

\section{Western blotting assay}

The CPM cellular proteins and muscle tissues were extracted using radio-immunoprecipitation assay (RIPA) buffer and phenylmenthanesulfonyl fluoride (PMSF) protease inhibitor. The Western blot assays were carried out as previously reported ${ }^{48}$. The antibodies used for Western blots were as follows: Flag tag polyclonal antibody (20543-1AP; Proteintech, USA; 1:1000), MyHC mouse monoclonal antibody (B103; DHSB, Lowa City, IA, USA; 1:500), rabbit anti-heavy chain Myosin (ab124205; Abcam, Cambridge, UK; 1:1000), HRP-conjugated monoclonal mouse antiglyceraldehyde-3-phosphate dehydrogenase (GAPDH) (KC5G5; Kangchen, China; 1:10,000). HRP conjugated goat anti-rabbit IgG (A21020; Abbkine, USA; 1:10,000) and HRP conjugated goat anti-mouse IgG (A21010; Abbkine, USA; $1: 10,000)$ were used as secondary antibodies.

\section{Immunofluorescence}

The CPM cells cultured in 12-well plates were treated with $4 \%$ formaldehyde for $20 \mathrm{~min}$ after $48 \mathrm{~h}$ of transfection. These experiments were carried out as previously reported $^{49}$. And images of immunofluorescence were captured with a Leica DMi8 fluorescent microscope (Leica, Wetzlar, Germany). The percentage of the total image area covered by myotubes as the total myotube area was calculated by using the Image J software (National Institutes of Health, Bethesda, MD, USA).

\section{RNA pull-down assay, mass spectrometry, and RNA immunoprecipitation}

The experiments were performed as previously reported $^{50}$.

\section{Lentiviral vector infection}

Three intramuscular injections of 1-day-old chicks were injected lentivirus into the gastrocnemius muscle at a dosage of $1 \times 10^{6} \mathrm{IU} / \mathrm{mL}$ (single dose injection started at Days 1,5 , and 9 ).

\section{Histology image analysis and immunohistochemical analysis}

Gastrocnemius muscle tissues of 14-day-old chicken were collected from LV-lncRNA-FKBP1C, LV-NC, LV-shRNAIncRNA-FKBP1C, and LV-shRNA-NC infected muscle ( $n=10 /$ case). After fixed with $4 \%$ paraformaldehyde, tissues 
were paraffin-embedded. Tissues sections were stained with Hematoxylin and Eosin(H\&E) and then subjected to image analysis. Also, Tissue sections were subjected to immunohistochemical analysis.

Primary antibodies used for immunohistochemistry were: Mouse anti-Myosin skeletal fast (MY-32, GeneTex, 1:400 dilutions on paraffin sections); Mouse anti-Myosin heavy chain, slow contracting muscle (S58, DSHB, 1:50 dilution on paraffin sections).

\section{Measurement of glycogen, lactate dehydrogenase (LDH) and succinate dehydrogenase (SDH)}

The chickens injected with lentivirus were fasted for $12 \mathrm{~h}$ before being sacrificed, and dissected gastrocnemius muscle. Glycogen, LDH and SDH were assayed using a Glycogen Content Assay Kit (Solarbio, BC0345), a Lacate Dehydrogenase (LDH) Activity Assay Kit (Solarbio, BC0685) and a Succinate Dehydrogenase (SDH) Activity Assay Kit (Solarbio, BC0955), according to the manufacturer's instructions.

\section{Statistical analysis}

All experimental results are presented as the mean $\pm \mathrm{S}$. E.M, based on at least three independent experiments. The statistical significance of differences between means was assessed by performing an unpaired Student's $t$-test. We considered $P<0.05$ to be statistically significant. ${ }^{*} P<$ $0.05 ;{ }^{* *} P<0.01 ;{ }^{* * *} P<0.001$.

\section{Acknowledgements}

This work was supported by the Natural Scientific Foundation of China (U1901206, 31802051 and 31761143014), Local Innovative and Research Teams Project of Guangdong Province (2019BT02N630), Ten-Thousand Talents Program (W03020593), and China Agriculture Research System (CARS-41-G03).

\begin{abstract}
Author details
'State Key Laboratory for Conservation and Utilization of Subtropical Agrobioresources \& Lingnan Guangdong Laboratory of Agriculture, College of Animal Science, South China Agricultural University, Guangzhou, Guangdong, China. ${ }^{2}$ Guangdong Provincial Key Lab of Agro-Animal Genomics and Molecular Breeding, and Key Laboratory of Chicken Genetics, Breeding and Reproduction, Ministry of Agriculture, Guangzhou, China. ${ }^{3}$ National-Local Joint Engineering Research Center for Livestock Breeding, Guangzhou, China
\end{abstract}

\section{Author contributions}

J.Y. and Z.W. contributed equally to this work. J.Y. designed the study, carried out all experiments, analyzed data, and wrote the paper. Z.W., X.Y. and M.M. participated in the design of the experiment and data analyses. Z.W. and Z.L. assisted with the manuscript's experimental discussion. Q.N. participated in the design, manuscript writing and final approval of the manuscript. All authors read and approved the final manuscript.

\section{Conflict of interest}

The authors declare no competing interests.

\section{Publisher's note}

Springer Nature remains neutral with regard to jurisdictional claims in published maps and institutional affiliations.
Supplementary information The online version contains supplementary material available at https://doi.org/10.1038/s41420-021-00463-7.

Received: 8 February 2021 Revised: 12 March 2021 Accepted: 25 March 2021

Published online: 09 April 2021

\section{References}

1. Frontera, W. R. \& Ochala, J. Skeletal muscle: a brief review of structure and function. Calcif. Tissue Int. 96, 183-195 (2015).

2. Li, R. et al. Exploring the IncRNAs related to skeletal muscle fiber types and meat quality traits in pigs. Genes 11, 883 (2020).

3. Fontelonga, T. M. et al. Sunitinib promotes myogenic regeneration and mitigates disease progression in the mdx mouse model of Duchenne muscular dystrophy. Hum. Mol. Genet. 28, 2120-2132 (2019).

4. Gordon, J. W., Dolinsky, V. W., Mughal, W., Gordon, G. R. \& McGavock, J. Targeting skeletal muscle mitochondria to prevent type 2 diabetes in youth. Biochem. Cell Biol. 93, 452-465 (2015).

5. Kim, S. N. \& Kim, J. Higher appendicular skeletal muscle mass protects metabolically healthy obese boys but not girls from cardiometabolic abnormality. Int. J. Environ. Res Public Health 16, 652 (2019).

6. Petchey, L. K. et al. Loss of Prox 1 in striated muscle causes slow to fast skeletal muscle fiber conversion and dilated cardiomyopathy. Proc. Natl Acad. Sci. USA 111, 9515-9520 (2014).

7. Boyer, J. G. et al. ERK1/2 signaling induces skeletal muscle slow fiber-type switching and reduces muscular dystrophy disease severity. JCI Insight 5, e127356 (2019).

8. Schiaffino, S. \& Reggiani, C. Molecular diversity of myofibrillar proteins: gene regulation and functional significance. Physiol. Rev. 76, 371-423 (1996).

9. Bottinelli, R. \& Reggiani, C. Human skeletal muscle fibres: molecular and functional diversity. Prog. Biophys. Mol. Biol. 73, 195-262 (2000).

10. Koutakis, P. et al. Abnormal accumulation of desmin in gastrocnemius myofibers of patients with peripheral artery disease: associations with altered myofiber morphology and density, mitochondrial dysfunction and impaired limb function. J. Histochem. Cytochem. 63, 256-269 (2015).

11. Shen, L. Y. et al. Effects of muscle fiber type on glycolytic potential and meat quality traits in different Tibetan pig muscles and their association with glycolysis-related gene expression. Genet. Mol. Res. 14, 14366-14378 (2015).

12. Choe, J. H. et al. The relation between glycogen, lactate content and muscle fiber type composition, and their influence on postmortem glycolytic rate and pork quality. Meat Sci. 80, 355-362 (2008).

13. Choe, J. H. \& Kim, B. C. Association of blood glucose, blood lactate, serum cortisol levels, muscle metabolites, muscle fiber type composition, and pork quality traits. Meat Sci. 97, 137-142 (2014).

14. Ma, M. et al. IncRNA-Six 1 is a target of miR-1611 that functions as a ceRNA to regulate Six 1 protein expression and fiber type switching in chicken myogenesis. Cells 7, 243 (2018)

15. Dou, M. et al. The long noncoding RNA MyHC $\| A X$ X-AS contributes to skeletal muscle myogenesis and maintains the fast fiber phenotype. J. Biol. Chem. 295 4937-4949 (2020)

16. Nagano, T. \& Fraser, P. Emerging similarities in epigenetic gene silencing by long noncoding RNAs. Mamm. Genome 20, 557-562 (2009).

17. Guttman, M. \& Rinn, J. L. Modular regulatory principles of large non-coding RNAs. Nature 482, 339-346 (2012).

18. Klattenhoff, C. A. et al. Braveheart, a long noncoding RNA required for cardiovascular lineage commitment. Cell 152, 570-583 (2013).

19. Kopp, F. \& Mendell, J. T. Functional classification and experimental dissection of long noncoding RNAs. Cell 172, 393-407 (2018).

20. Shi, X., Sun, M., Liu, H., Yao, Y. \& Song, Y. Long non-coding RNAs: a new frontier in the study of human diseases. Cancer Lett. 339, 159-166 (2013).

21. Ponting, C. P., Oliver, P. L. \& Reik, W. Evolution and functions of long noncoding RNAs. Cell 136, 629-641 (2009).

22. Li, Z. et al. LncIRS1 controls muscle atrophy via sponging miR-15 family to activate IGF1-PI3K/AKT pathway. J. Cachexia Sarcopenia Muscle 10, 391-410 (2019).

23. Cai, B. et al. LncRNA-Six 1 encodes a micropeptide to activate Six 1 in Cis and is involved in cell proliferation and muscle growth. Front. Physiol. 8, 230 (2017). 
24. Jin, J. J. et al. Long noncoding RNA SYISL regulates myogenesis by interacting with polycomb repressive complex 2. Proc. Natl Acad. Sci. USA $\mathbf{1 1 5}$ E9802-E9811 (2018).

25. Zhou, J. et al. H19 IncRNA alters DNA methylation genome wide by regulating S-adenosylhomocysteine hydrolase. Nat. Commun. 6, 10221 (2015).

26. Wang, $L$. et al. LncRNA Dum interacts with Dnmts to regulate Dppa2 expression during myogenic differentiation and muscle regeneration. Cell Res. 25, 335-350 (2015).

27. Ulitsky, I. \& Bartel, D. P. lincRNAs: genomics, evolution, and mechanisms. Cell 154, 26-46 (2013).

28. Ma, M. Z. et al. Long noncoding RNA GCASPC, a target of miR-17-3p, negatively regulates pyruvate carboxylase-dependent Cell proliferation in gallbladder cancer. Cancer Res. 76, 5361-5371 (2016).

29. Whalen, R. G., Schwartz, K., Bouveret, P., Sell, S. M. \& Gros, F. Contractile protein isozymes in muscle development: identification of an embryonic form of myosin heavy chain. Proc. Natl Acad. Sci. USA 76, 5197-5201 (1979).

30. Chong, J. X. et al. Autosomal-dominant multiple pterygium syndrome is caused by mutations in MYH3. Am. J. Hum. Genet. 96, 841-849 (2015).

31. Toydemir, R. M. et al. Mutations in embryonic myosin heavy chain (MYH3) cause Freeman-Sheldon syndrome and Sheldon-Hall syndrome. Nat. Genet. 38, 561-565 (2006).

32. Cho, I. C. et al. A functional regulatory variant of MYH3 influences muscle fibertype composition and intramuscular fat content in pigs. PLOS Genet. 15, e1008279 (2019).

33. Cesana, M. et al. A long noncoding RNA controls muscle differentiation by functioning as a competing endogenous RNA. Cell 147, 358-369 (2011).

34. Zhu, M. et al. Lnc-mg is a long non-coding RNA that promotes myogenesis. Nat. Commun. 8, 14718 (2017).

35. Wang, S. et al. Long noncoding RNA Neat1 modulates myogenesis by recruiting Ezh2. Cell Death Dis. 10, 505 (2019).

36. Zhang, Z. K. et al. A newly identified IncRNA MAR1 acts as a miR-487b sponge to promote skeletal muscle differentiation and regeneration. J. Cachexia Sarcopenia Muscle 9, 613-626 (2018).

37. Kallen, A. N. et al. The imprinted H19 IncRNA antagonizes let-7 microRNAs. Mol. Cell 52, 101-112 (2013)
38. Dey, B. K., Pfeifer, K. \& Dutta, A. The H19 long noncoding RNA gives rise to microRNAs miR-675-3p and miR-675-5p to promote skeletal muscle differentiation and regeneration. Genes Dev. 28, 491-501 (2014).

39. Wu, F. et al. Effect of skeletal muscle fibers on porcine meat quality at different stages of growth. Genet. Mol. Res. 14, 7873-7882 (2015).

40. Ismail, I. \& Joo, S. T. Poultry meat quality in relation to muscle growth and muscle fiber characteristics. Korean J. Food Sci. Anim. Resour. 37, 873-883 (2017).

41. Ryu, Y. C. \& Kim, B. C. The relationship between muscle fiber characteristics, postmortem metabolic rate, and meat quality of pig longissimus dorsi muscle. Meat Sci. 71, 351-357 (2005)

42. Lee, $\mathrm{S}$. et al. Noncoding RNA NORAD regulates genomic stability by sequestering PUMILIO proteins. Cell 164, 69-80 (2016).

43. Tichon, A. et al. A conserved abundant cytoplasmic long noncoding RNA modulates repression by Pumilio proteins in human cells. Nat. Commun. 7, 12209 (2016).

44. Cameron-Christie, S. R. et al. Recessive spondylocarpotarsal synostosis syndrome due to compound heterozygosity for variants in MYH3. Am. J. Hum Genet. 105, 669 (2019).

45. Scala, M. et al. A novel pathogenic MYH3 mutation in a child with SheldonHall syndrome and vertebral fusions. Am. J. Med. Genet. A 176, 663-667 (2018).

46. Maran, S. et al. Mutations in the tail domain of MYH3 contributes to atria septal defect. PLoS ONE 15, e230982 (2020).

47. Livak, K. J. \& Schmittgen, T. D. Analysis of relative gene expression data using real-time quantitative PCR and the 2(-Delta Delta C(T)) method. Methods $\mathbf{2 5}$ 402-408 (2001)

48. Bass, J. J. et al. An overview of technical considerations for Western blotting applications to physiological research. Scand. J. Med. Sci. Sports 27, 4-25 (2017).

49. Wang, Z. et al. MiR-34b-5p mediates the proliferation and differentiation of myoblasts by targeting IGFBP2. Cells 8, 360 (2019).

50. Li, Z. et al. The long noncoding RNA THRIL regulates TNFalpha expression through its interaction with hnRNPL. Proc. Natl Acad. Sci. USA 111, 1002-1007 (2014). 